\title{
Rapidly progressive amnestic syndrome with psychiatric and autonomic features: extending the spectrum of GFAP-astrocytopathies and autoimmune dementias?
}

\section{Maximilian Friedrich ( $\nabla$ Friedrich_M6@ukw.de)}

University Hospital Wuerzburg Department of Neurology https://orcid.org/0000-0002-3695-1268

Johannes Hartig

University Hospital Wurzburg: Universitatsklinikum Wurzburg

Harald Prüß

Charité Universitätsmedizin Berlin: Charite Universitatsmedizin Berlin

Chi Wang Ip

University Hospital Wurzburg: Universitatsklinikum Wurzburg

Jens Volkmann

University Hospital Wurzburg: Universitatsklinikum Wurzburg

\section{Research Article}

Keywords: Neuroimmunology, Dementia, Neuroinflammation, Encephalitis

Posted Date: July 28th, 2021

DOI: https://doi.org/10.21203/rs.3.rs-752817/v1

License: (c) (i) This work is licensed under a Creative Commons Attribution 4.0 International License.

Read Full License

Version of Record: A version of this preprint was published at Annals of Clinical and Translational Neurology on February 7th, 2022. See the published version at https://doi.org/10.1002/acn3.51513. 


\section{Abstract}

Objective Autoimmune glial fibrillary acidic protein astrocytopathy (GFAP-A) is a steroid-responsive meningoencephalomyelitis, sometimes presenting with atypical clinical signs such as movement disorders or psychiatric and autonomic features. Diagnosis relies on clinical presentation, detection of GFAP autoantibodies in CSF as well as ancillary MRI biomarkers. We aimed to characterize two patients seropositive for GFAP antibodies in CSF presenting with a highly atypical clinical picture of isolated neurocognitive and autonomic features resembling neurodegenerative dementia in absence of cranial MRI abnormalities.

Methods Semiquantitative, clinicoradiological, serologic and immunohistochemical analysis of two cases with longitudinal observation over a time course of 18-24 months.

Results Both patients had rapidly progressive amnestic syndromes in absence of other focal neurologic signs. Cranial MRI was grossly unremarkable while functional neuroimaging showed cortical and subcortical metabolic changes. Tissue-based assays (TBA) of CSF confirmed GFAP-specific astrocytic binding patterns in rat corpus callosum and hippocampus in both cases. Incomplete steroid response prompted therapy escalation to B-cell depletion, which improved or stabilized disease courses over the observation time.

Conclusions These cases suggest that rapidly progressive amnestic syndromes with psychiatric and autonomic features may be a novel phenotype of GFAP astrocytopathy as well as a potentially underrecognized mimic of neurodegenerative dementia amenable to immunomodulation. We recommend including GFAP-AB screening using TBA in the work-up of rapidly progressive dementias especially when autoimmune etiologies are suspected.

\section{Introduction}

Glial fibrillary acidic protein (GFAP) is an astrocytic intermediate filament with manifold pathophysiological implications. In 2016, a novel astrocytopathy associated with GFAP-antibodies (AB), extending the nosologic spectrum defined by Aquaporin-4-AB (AqP4-AB) mediated astrocytopathy, Neuromyelitis Optica (NMO) ${ }^{1}$. GFAP autoimmunity (GFAP-A) usually manifests after an unspecific, often viral prodrome as an acute to subacute meningoencephalomyelitis or isolated subforms thereof, with monophasic or relapsing course ${ }^{2}$. GFAP-A etiology ranges from paraneoplastic to parainfectious and idiopathic ${ }^{1}$. Remarkably, GFAP-A is frequently embedded in a context of overlapping autoimmunity, raising the question of an emerging clinicopathological spectrum ${ }^{3}$. In the limited number of neuropathological investigations to date, GFAP-A was shown to be associated with microglial activation and perivascular inflammation sparing vascular walls ${ }^{3}$.

Diagnosis of GFAP-A relies on detection of GFAP-autoantibodies in CSF, since their presence in serum is less specific ${ }^{4}$. In tissue-based assays (TBA), the IgG staining pattern is mostly filamentous appearing and 
adjacent to pial, subpial and subependymal portions of the CNS ${ }^{4}$. MRI shows subcortical white and grey matter abnormalities in $~ 50-60 \%$ and myelitic changes in $\sim 44 \%$ of patients. Moreover, subcortical radial perivascular contrast enhancement is commonly found ${ }^{3}$. Cranial ${ }^{18}$ Fluor-Desoxy-Glucose Positron Emission Tomography (FDG-PET) in single GFAP-A cases has shown both subcortical hypometabolism and cortical hypemetabolism ${ }^{4}$. Monophasic GFAP-A typically shows favorable response to corticosteroids, however, up to $50 \%$ of cases are steroid-dependent or relapsing, requiring escalation of immunotherapy ${ }^{1}$.

\section{Case Reports: Methods And Results}

Both patients presented around their 7th decade with abrupt onset and rapidly progressive neurocognitive deficits accompanied by autonomic and psychiatric abnormalities. Focal neurologic signs or significant cranial MRI abnormalities were absent. GFAP-AB were detected by tissue-based assays (TBA) and confirmed by cell-based assays (CBA) in CSF (synopsis see Table 1 \& supplementary table 2). 
Table 1

Synopsis of clinical findings.

\section{Patient 1}

Initial symptoms

Vegetative

Symptoms
Intermittent disorientation and navigational problems. Short term memory deficits and practical deficits
Patient 2

Intermittent disorientation, hallucinations, avolition, impairment of long-term and episodic more than short-term memory

Insomnia, diurnal somnolence, weight loss due to anorexia, constipation, meteorism, reduced libido and erectile dysfunction

\section{Psychiatric Symptoms}

Concomitant diagnoses

Neurological examination

Neuropsychological assessment (CERAD-Plus)
Insomnia, night sweats, weight loss and anorexia, hyposmia, chronic constipation, asthenia
Major depressive disorder, SIADH, arterial hypertension, hypogonadotropic hypogonadism
Major depressive disorder, chronic constipation, left bundle branch block, meningioma
Anxiety, Depression, Avolition

Disoriented to time. No other abnormalities.
Imapairments in semantic $(z=-2.1)$ and phonematic fluency $(z=-1.6)$, word finding $(z=-1.8)$, performance $(z=-3.1)$, visuoconstruction $(z=-3.2)$, visual memory $(z=-2.9)$. Visuomotor slowdown $(z=-2,9)$. The Frontal Assessment Battery (FAB) moderate frontal-executive deficits. No evidence of depressiveness ( 9 points with a cut-off of 11), mild anxiety symptomatology (11 with cut-off at 11).

Improvements in phonematic fluency ( $\mathrm{z}$ $=-0.84)$. Deterioration in cognitive flexibility. All other domains stable. No depressive symptoms ( 9 points with a cut-off of 11) or pathological anxiety symptoms (6 points with cut-off at 11).
Impairments in learning performance $(z=-2.7)$, consolidation performance $(z=-$ $4.0)$, recognition performance $(z=$ $-4.6)$, intrusion $(z=-2.9)$, naming performance $(z=-1.9)$, semantic and phonematic fluency $(z=-3.5$ and $z=-3.3)$, figural memory $(z=$ - 2.7), visuoconstruction ( $z=$ -2.9), visuomotor speed, sustained attention $(z=-2.7)$

CERAD-Plus Improvements in consolidation performance $(\mathrm{z}=-$ $1.2)$, recognition $(z=0.81)$ intrusion $(z=0.68)$, naming $(z=$ $-0.49)$, semantic fluency $(z=$ -0.79), phonematic fluency $(z=$ $0.52)$, visuoconstruction $(z=$ $0.75)$, visuomotor speed $(z=$ -0.64), sustained attention $(z=$ $0.16)$.

\section{Patient 1}

This patient complained of disorientation and memory loss accompanied by an anxious depression, insomnia and constipation over the preceding six months. Neuropsychological assessment revealed impairments especially in linguistic, visuoconstructive and executive domains $(z=-3.2$ to -1.8$)$. Serum antineuronal antibody panel (ANP) detected GFAP-IgG (titre 1:1000, CBA). Cranial FDG-PET disclosed 
fronto-parieto-occipital hypometabolism. CSF analysis revealed elevated protein levels and marginally decreased ß-Amyloid-42. Intravenous methylprednisolone pulse therapy (IVMP, 1000mg/d for three days) was administered. At five-month follow-up, neurocognitive deficits persisted, hence, five sessions of plasmapheresis and Rituximab $1000 \mathrm{mg}$ every six months were initiated. At 1.5-year follow-up, linguistic deficits had markedly improved ( $z=-1.6$ vs. $z=-0.84)$ and cognitive flexibility worsened while all other domains remained stable $(z=-3.4$ to -0.84$)$.

\section{Patient 2}

This patient reported disorientation, hallucinations and long-term memory impairment, accompanied by anxious depression, insomnia and reduced libido. Neuropsychological assessment revealed severe impairments ( $z=-4.6$ to -2$)$ in most domains. Blood work showed moderate hyponatremia consistent with SIADH and hypogonadotropic hypogonadism. Serum ANP was negative and isolated antinuclear antibodies detectable (titre 1:80). Cranial FDG-PET showed cortical hypometabolism besides striatal hypermetabolism. CSF showed lymphocytic pleocytosis $\left(28 / \mathrm{mm}^{3}\right)$. IVMP $(100 \mathrm{mg} / \mathrm{d}$ for three days) followed by five sessions of immunoadsorption and steroid taper were initiated. Incomplete remission and persisting pleocytosis $\left(15 / \mathrm{mm}^{3}\right)$ at 3-month follow up prompted initiation of Rituximab $1000 \mathrm{mg}$. Two years later, all neuropsychological results were within normal limits $(z=-1.2$ to +0.81$)$.

After initial treatment, GFAP-AB were undetectable in serum of patient 1 (CSF unavailable) and serum \& CSF of patient 2. Repeat FDG-PET revealed no neoplasms in both.

\section{Discussion}

These cases are highly atypical for GFAP-A not only in that they lack typical clinicoradiological features, but also in that the clinical picture which was dominated by a rapidly progressive dementia with psychiatric and autonomic features. Although the clinical phenotype described in GFAP-A case series has successively been extended ${ }^{5}$, the given presentations of GFAP-A have, to the best of our knowledge, not yet been reported. Progressive neurocognitive deficits resembling neurodegenerative dementia in GFAP-A are in line with growing evidence for astroglial dysfunction in neuropsychiatric conditions ${ }^{6}$. Adding to this, accompanying encephalopathic signs are reported in up to $60 \%$ of GFAP-A cases, but are unspecific in nature ${ }^{4}$. Experimental evidence suggesting differential CNS tropisms of GFAP autoimmunity points toward dissociable phenotypes, acute meningitic-vasculitic and chronic relapsing encephalitic ${ }^{7}$. Our observation may support the notion of a further evolving clinical spectrum of GFAP-A and of a potentially emerging chronic encephalitic subtype presenting as progressive dementia.

Our observations further suggest that GFAP-AB may be a valuable addition to autoimmune dementia panels ${ }^{8}$, especially since given cases mimic typical neurodegenerative dementia and may therefore go unrecognized and worse, untreated. Especially patient 1 showed features strongly suggestive of neurodegenerative dementia. However, detection of CSF GFAP-AB and cognitive stabilization over 1.5 years associated with immunotherapy demonstrate a relevant, perhaps secondary autoimmunological 
component amenable to immunomodulation. In fact, this bears resemblance with IgLON5-disease as the prototype of overlapping autoimmunity and neurodegeneration ${ }^{9}$, mostly presenting with sleep related, autonomic and neuropsychiatric symptoms, like the syndromes described here. Insomnia, neurohumoral and autonomic features all map to the diencephalon, a somewhat pathognomonic lesion site in AQP4-AB astrocytopathy, IgLON5-disease and, less frequently, also in GFAP-A ${ }^{4,5}$. However, with C-MRI being unremarkable in both, functional rather than structural diencephalic deficits, ought to be postulated.

In contrast to IgLON5-disease however, we observed lasting and quantifiable effects of intensified immunotherapy in our patients and therefore argue that GFAP-AB may serve as valuable biomarker to identify immunotherapy-responsive forms of cognitive impairment driven by astroglial inflammation. After all, identifying treatment potential in devastating neurocognitive deficits is pivotal from a clinical perspective.

\section{Declarations}

Ethics approval and consent to participate and disclose:

Patients gave informed written consent to consent, disclose and for publication of this retrospective study.

Data availability:

Non-identifiable data is available upon reasonable request to the corresponding author.

Competing interests:

Authors declare no conflicts of interest pertaining to the manuscript.

Funding:

Dr. Maximilian U. Friedrich receives funding from the Interdisciplinary Center for Clinical Research (IZKF) Z2-CSP13 at the University Hospital Wuerzburg.

\section{Authors' contributions:}

MF obtained and analyzed patient data, conceptualized, drafted and revised the manuscript. JH analyzed patient data and drafted the manuscript. HP analyzed patient data and revised the manuscript. CWI and JV obtained patient data and revised the manuscript.

\section{Acknowledgements:}

Not applicable

\section{References}


1. Fang B et al (2016) Autoimmune glial fibrillary acidic protein astrocytopathy: A novel meningoencephalomyelitis. JAMA Neurology 73:1297-1307

2. Flanagan EP et al (2017) Glial fibrillary acidic protein immunoglobulin $\mathrm{G}$ as biomarker of autoimmune astrocytopathy: Analysis of 102 patients. Ann Neurol 81:298-309

3. Shan F, Long Y, Qiu W (2018) Autoimmune Glial Fibrillary Acidic Protein Astrocytopathy: A Review of the Literature. Front Immunol 0:2802

4. Kunchok A, Zekeridou A, McKeon A (2019) Autoimmune glial fibrillary acidic protein astrocytopathy. Curr Opin Neurol 32:452-458

5. Kimura A, Takekoshi A, Yoshikura N, Hayashi Y, Shimohata T (2019) Clinical characteristics of autoimmune GFAP astrocytopathy. J Neuroimmunol 332:91-98

6. Middeldorp J, Hol EM (2011) GFAP in health and disease. Prog Neurobiol 93:421-443

7. Sasaki K et al (2014) Relapsing-Remitting Central Nervous System Autoimmunity Mediated by GFAP-Specific CD8 T Cells. J Immunol 192:3029-3042

8. Long JM, Day GS (2018) Autoimmune Dementia. Semin Neurol 38:303-315

9. Heidbreder A, Philipp K Anti-IgLON 5 Disease. Current Treatment Options in Neurology vol. 20 (2018)

\section{Figures}



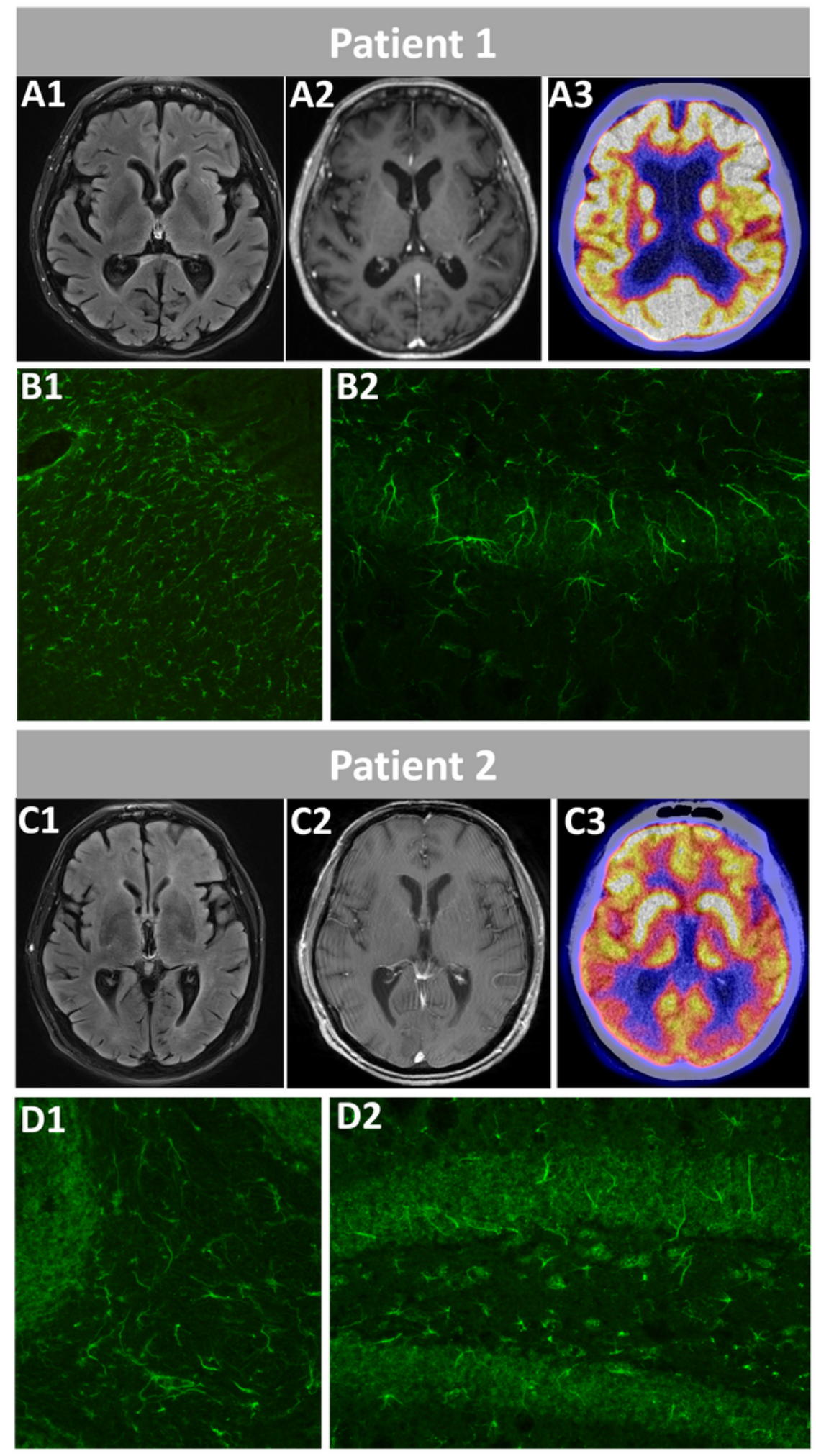

\section{Figure 1}

Clinical and molecular imaging findings. Patient 1: A1-2, representative axial C-MRI images in T2w and T1w-Gd techniques disclose no parenchymal, vascular or blood-brain barrier abnormalities. A3, axial FDGPET overlayed on computed tomography (CT) showing subtle left occipital hypometabolism with otherwise normal cortical and subcortical tracer uptake. B, indirect immunofluorescence (IF) using patient CSF on mouse corpus callosum (B1) and hippocampus (B2) slices. Both images show the characteristic 
filamentous, astrocytic staining pattern of GFAP-AB. Scale bar $=100 \mu \mathrm{m}$. Patient 2: C1-2, representative axial C-MRI images in T2w and T1w-Gd techniques showing mild microangiopathic white matter hyperintensities but no further parenchymal, vascular or blood-brain barrier abnormalities. C3, axial FDGPET overlayed on computed tomography (CT) showing left hemispheric dominant occipito-temporal hypometabolism and pronounced striatal hypermetabolism. D, Both images show the characteristic astrocytic staining pattern of GFAP-AB (methods see B, scale bar $=100 \mu \mathrm{m}$ ). 